\title{
»Ein persönlicher Charakter ist schon deshalb ein sozialer, weil er sich nur so ausbilden kann « Wolf Rainer Wendt ist 70
}

\author{
Günter Rieger
}

Wolf Rainer Wendt feierte am 14. Mai 2009 seinen 70. Geburtstag. Seiner Person gilt es zu gratulieren. Sein Werk gilt es zu würdigen.

Wendt wurde als Person wie in seinem Werk durch die Zeitumstände geprägt, manchmal gebeutelt, stets herausgefordert und hat doch zugleich, durch ein hohes Maß an Disziplin, Augenmaß und Eigenständigkeit, die Umstände gestaltet, hat sich selbst als Person geformt und ein Werk mit weitreichendem Einfluss auf die Soziale Arbeit geschaffen.

Während seine ersten Lebensjahrzehnte turbulent wirken, erscheint er in späteren Jahren als umsichtiger Herr der eigenen Lebensführung. Stets haushaltend mit den eigenen Ressourcen, konzentriert, effizient und effektiv mit Blick auf die Sache der Sozialen Arbeit und das eigene Werk, organisiert er berufliches wie privates Leben. Ein vorbildliches, wirksames Selbstmanagement. Dorthin aber führt zunächst ein verschlungener Weg prägender Lebensstationen.

\section{Jugendjahre 1939 bis 1958}

Als Wolf Rainer Wendt 1963 im schwäbischen Tübingen ein Studium begann, lagen - zeitgeschichtlich bedingt wie persönlich gefärbt - einschneidende Lebenserfahrungen hinter ihm. Kindheit und Jugend waren geprägt durch die beiden deutschen Diktaturen. Geboren 1939 in Schwerin in Mecklenburg erlebte er als Kind die Endphase des Nationalsozialismus, die Schrecken des Krieges und die

Prof. Dr. Günter Rieger ist Dekan des Studienbereichs Sozialwesen an der Dualen Hochschule BadenWürttemberg in Stuttgart. E-Mail rieger@ba-stuttgart.de
Wirren der Nachkriegszeit. Die Jugendzeit fiel in die Phase der Gründerjahre der DDR. Wendt engagierte sich - wie man es erwartete - politisch ("Junge Pioniere ", »Kader der FDJ «), passte sich an, kritisierte, eckte an, landete 1957 als politischer Häftling für über ein Jahr im Zuchthaus. Seine schulische (Oberschule, Abendgymnasium) und berufliche Ausbildung (Chemiker) bleiben unabgeschlossen. Ohne Perspektive entlassen, floh er 1958 über West-Berlin aus der DDR.

\section{Studienjahre 1963 bis 1969}

Nach seiner Übersiedlung in die Bundesrepublik und einer eher unsteten Orientierungsphase, in der er mit "manchen Mühen « das Abitur nachholte, begann er 1963 ein Studium der Psychologie und Philosophie an der EberhardKarls-Universität Tübingen - einer altehrwürdigen Universität, eingebettet in ein schwäbisch kleinstädtisches Milieu und beeinflusst durch den sich abzeichnenden Wandel und Aufbruch der 1960er-Jahre. Ernst Bloch, Walter Schulz, Ralf Dahrendorf und Walter Jens waren prägende Persönlichkeiten jener Zeit. Im Jahre 1968 erwarb Wendt einen
Abschluss als Diplompsychologe und stellte seine Dissertation in Philosophie über Marcel Duchamp bei Otto Fiedrich Bollnow fertig; ein Jahr später erfolgte die Promotion.

\section{Praxisjahre 1969 bis 1977}

Im Jahre 1969 verließ Wolf Rainer Wendt die Universität, um eine Stelle beim Jugendamt der Stadt Stuttgart anzunehmen. In der Erziehungsberatung fand er eine ihm gemäße Nische im Grenzgebiet von Psychologie und Sozialpädagogik. Er spezialisierte sich auf die Begutachtung von Kleinkindern aus Heimen, vor Adoptionen etc. und übernahm 1970 eine neu geschaffene Stelle mit Zuständigkeit für die Stuttgarter Heime. Schließlich leitete er seit 1972 die neu gebildete Abteilung »Sozialpädagogische Heime, Adoptions- und Pflegestellenwesen «. Mit seinem Tätigkeitsbereich war Wendt in Aufbau und Umgestaltung der Jugendhilfe einer deutschen Großstadt in einer für die heutige Gestalt der Jugendhilfe entscheidenden Reformphase involviert. Er beteiligte sich am fachlichen Diskurs und verwirklichte innovative Projekte (Kurkliniken für Kinder; Pflegekinderwesen; Tagesmütter), die er stets

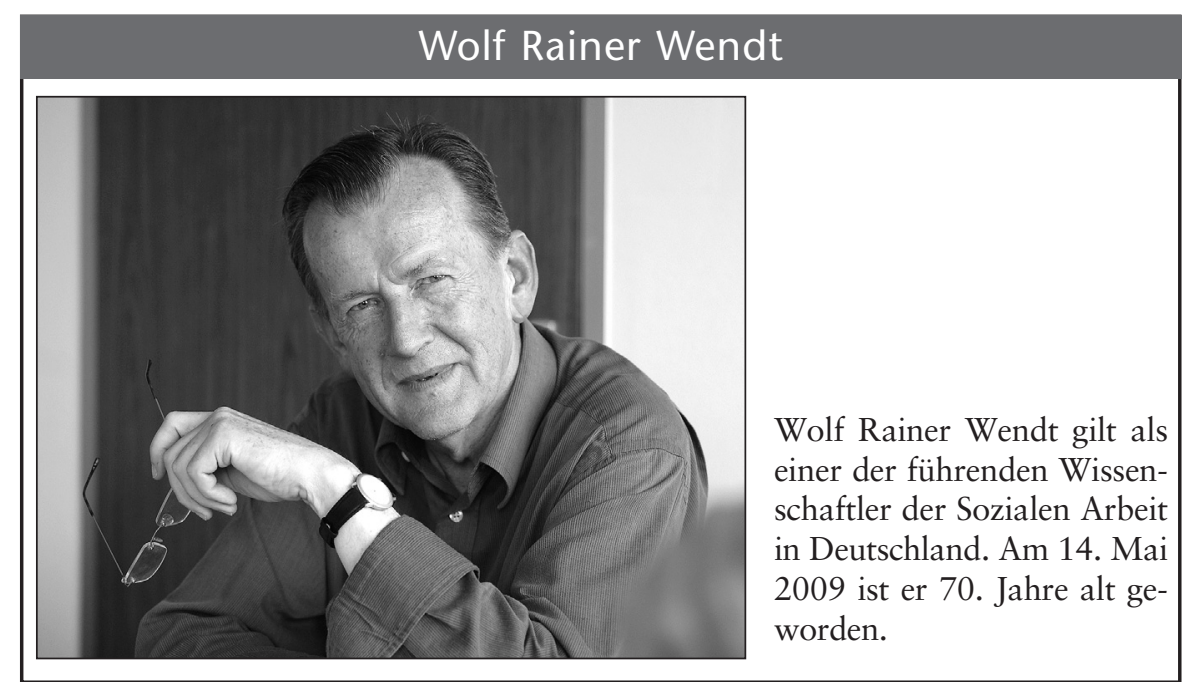


unmittelbar wissenschaftlich reflektierte und publizierte.

\section{Akademikerleben 1977 bis heute}

Im Jahre 1977 erfolgte der Wechsel zurück in die Lehre. Wendt übernahm die Leitung des neu eingerichteten Bereichs Sozialwesen an der Berufsakademie Stuttgart. Von Anfang an war er als Berater und nebenamtlicher Dozent an der Entwicklung des neuen Studienangebots beteiligt. Unter seiner Leitung entstand aus bescheidenen Anfängen ein anerkannter Diplomstudiengang Sozialpädagogik/Sozialarbeit mit heute über 600 eingeschriebenen Studierenden. Neben der Gesamtleitung des Bereichs behielt Wendt über die Zeit die Zuständigkeit für die Fachrichtung Erziehungshilfen und Heimerziehung. Notwendigkeiten der Lehre, wissenschaftliche Interessen wie praktische Erfahrungen führten in den folgenden Jahren zu einer reichen Publikationsund Forschungstätigkeit. Ort und Ziel des Schaffens war dabei nie der akademische Elfenturm. Stets ging es um die Verknüpfung unterschiedlicher Disziplinen und die Verbindung von Theorie und Praxis. In der kontinuierlichen Ausweitung seiner Forschungsgebiete erwies er sich als transdisziplinärer Grenzgänger und hartnäckiger Propagandist für die Entwicklung der Sozialen Arbeit als Disziplin und Profession. Den von ihm für wichtig erkannten Inhalten versuchte er immer auch professionspolitisch Wirkung zu verschaffen. Dass er 2004 emeritiert wurde, entband ihn von seinen Leitungsaufgaben an der Berufsakademie Stuttgart, bedeutete aber keinen Bruch in seinem Engagement für Profession und Disziplin Sozialer Arbeit. Er war Vorsitzender der Deutschen Gesellschaft für Soziale Arbeit (die er 1989 mit gegründet hat) und ist es noch bei der Deutschen Gesellschaft für Care und Case Management, engagiert sich im Beirat verschiedener Fachzeitschriften (Blätter der Wohlfahrtspflege, SOZIALwirtschaft, SOZIALwirtschaft aktuell), entfaltet eine umfangreiche Vortragstätigkeit, bleibt in der Lehre aktiv (seit 2003 auch als Honorarprofessor am Institut für Erziehungswissenschaften der Universität Tübingen) und publiziert mit ungebrochenem Elan.

\section{Das Werk}

Auch nur einen halbwegs vollständigen Überblick über Wendts Werk zu präsentieren, sprengt jeden Rahmen. Dies gilt nicht nur für die außerordentliche Zahl an Monografien, Herausgeberschaften und Aufsätzen. Dies gilt vor allem für die bearbeitete Vielfalt der Themen (von der Sozialarbeitswissenschaft über die Sozialarbeitsforschung, die Sozialinformatik und die klinische Sozialarbeit, das Case Management, das Sozialmanagement, die Sozialwirtschaft, Fachsozialarbeit usw. bis hin zu Zivilgesellschaft und Ethik). Kaum ein relevantes Thema der Sozialen Arbeit in den letzten Jahrzehnten, zu dem er nicht publiziert und Stellung genommen hätte. Stets am Puls der Zeit hat er ein untrügliches Gespür für die Bedeutung neuer Phänomene und kommender Herausforderungen. Schnell und präzise, immer neue Verbindungen knüpfend, vertieft er sich in eine Thematik, um sie dann eigenständig aber auch eigenwillig zu verarbeiten. Viele Themen hat er als einer der Ersten aufgegriffen, hat die fachliche Diskussion angestoßen. Dabei sind die Themenauswahl und vor allem ihre Verarbeitung keineswegs beliebig. Wendt hat sich durch seine historischen wie sozialarbeitstheoretischen Forschungen einen stabilen wissenschaftlichen Bezugsrahmen geschaffen, der seine Wahrnehmung schärft und die ebenso innovative wie kreative Reflexion der Themen ermöglicht.

Wendt sah früh die Notwendigkeit, dass Soziale Arbeit über eine eigenständige Theorie integriert werden muss. Herausfordernd nahm er den Mangel an orientierenden Konzepten und das Fehlen eines Bezugsrahmens für Disziplin und Profession Sozialer Arbeit wahr. Ende der 1970er Jahre begann er mit der Arbeit an einer ökosozialen Theorie Sozialer Arbeit. Es entstand »Ökologie und Soziale Arbeit « (1982), dem Jahre später ein zweiter, weiterentwickelter Entwurf »Ökosozial denken und handeln « (1990) folgte.

Wendts Theorie gestaltete sich vor einem gesellschaftspolitischen Hintergrund, in dem die ökologische Frage immer drängender wurde und die Wissenschaft ökologischen Zusammenhängen aus systemtheoretischer Perspektive größere Aufmerksamkeit widmete. Im angelsächsischen Sprachraum machten insbesondere Carol B. Germain und Alex
Gitterman die ökologische Perspektive für die Soziale Arbeit fruchtbar. Deren "Life Model « aber blieb ein stark handlungsorientiertes Lehrbuch für die sozialarbeiterische Praxis mit eher geringer theoretischer Fundierung. Wendt nutzte die ihm zugänglichen vielfältigen geschichtlichen, philosophischen und psychologischen Bezüge um eine theoretisch anspruchsvolle ökosoziale Handlungstheorie $\mathrm{zu}$ formen.

Ebenso zentral wie innovativ ist dabei die von ihm in diesen Zusammenhang eingefügte Denkfigur des »Haushalts «. Die ökologische Perspektive der Wechselwirkung zwischen Individuum und Umwelt, ihre Auswirkung für die persönliche Lebensgestaltung wie das soziale Leben werden am Modell des »Haushalts « verstehbar. "Die ökosoziale Theorie wählt den >Haushalt $<$ zu seiner zentralen Kategorie, weil dieser Grundbegriff die Gestaltung und Bewältigung des Alltags im einzelnen an den Ertrag im ganzen Leben, an gesellschaftliche Kooperation und Verantwortung für die Natur im Haushalt der Schöpfung knüpft." (Wendt 1990: S. 29) Orientiert und organisiert in dieser für die Theorie zentralen Denkfigur gelingt es über Begriffe wie ökologische Bilanzierung, Lebenslagen, Nischen, Selbstorganisation, Ressourcen, Lebensbewältigung, Unterstützung, Vernetzung usw. einen verlässlichen Bezugsrahmen zur Reflexion sozialer Probleme wie sozialarbeiterischer/sozialpädagogischer Praxis zu schaffen.

Ökosozial liegt es dann auch nahe, die Notwendigkeit wirtschaftlichen Handelns für die Soziale Arbeit zu erkennen. Der an der Denkfigur des Haushalts geschärfte ökosoziale Blick ließ Wendt sehr früh die Bedeutung ökonomischen Denkens für die Soziale Arbeit erkennen. Wendt systematisiert Sozialwirtschaft über ihre eigenständige Zwecksetzung, ihre solidarische Organisationsform wie ihre wirtschaftliche Bedeutung und Notwendigkeiten. »Im Prozess der gesellschaftlichen Lebensführung ist im Hinblick auf die Chancen der Entwicklung, Teilhabe und Problembewältigung ..., eine vielgestaltige Soziale Arbeit zu leisten, und es sind Arrangements zu treffen, in denen diese Arbeit einen nachhaltigen Erfolg verspricht. Die Knappheit der Ressourcen, die dafür eingesetzt werden, verlangt eine angemessene Bewirtschaftung. " (Wendt 2008: S. 955 f.) Soziale 
Arbeit darf, gerade wenn sie bedarfsorientiert wirken will, die (sozial)wirtschaftlichen Zusammenhänge nicht vernachlässigen und muss sich den Instrumentenkoffer des (Sozial-)Managements erschließen.

Folgerichtig erscheint da auch Wendts methodische Affinität zum Case Management. Eine ökosoziale Theorie, die über das Modell des Haushalts organisiert ist, verlangt eine Methode, die ressourcenorientiert, systematisch planend, kontrolliert und kontrollierend ist, die selbstständiges Handeln mit Unterstützungsstrukturen verknüpft. Wendt war einer der ersten, der diese Methode aufgreift und in Deutschland propagiert. Case Management gilt ihm als effektive und effiziente Methode zur Steuerung einzelfallbezogener Leistungserbringung. Sie ermöglicht die professionelle Organisation von Hilfe vom Intake, über Assessment, Planning, Implementation bis hin zur Evaluation. Nutzerbezogen und ressourcenorientiert geht es darum, mit und für den Klienten ein persönliches wie professionelles Unterstützungsnetzwerk zu knüpfen, das dem Klienten eine gelingendere Lebensbewältigung ermöglicht. Dabei verteidigt Wendt das Case Management aus ökosozialer Perspektive gegen individualistische Fehlinterpretationen. Für ihn steht »case « nicht für den Menschen, »sondern für die problematische Situation, die es - im Ganzen und im Detail - zu bewältigen gilt. Sie sist der Fall und Gegenstand der ziel- und lösungsorientierten professionellen Bemühung. «(Wendt 2008: S. 174) Sie gilt es unter Aktivierung der Selbsthil$\mathrm{fe}$ und des Bewältigungsverhaltens des Klienten, seines sozialen Umfeldes und

\section{Soziale Arbeit in Wissenschaft und Praxis}

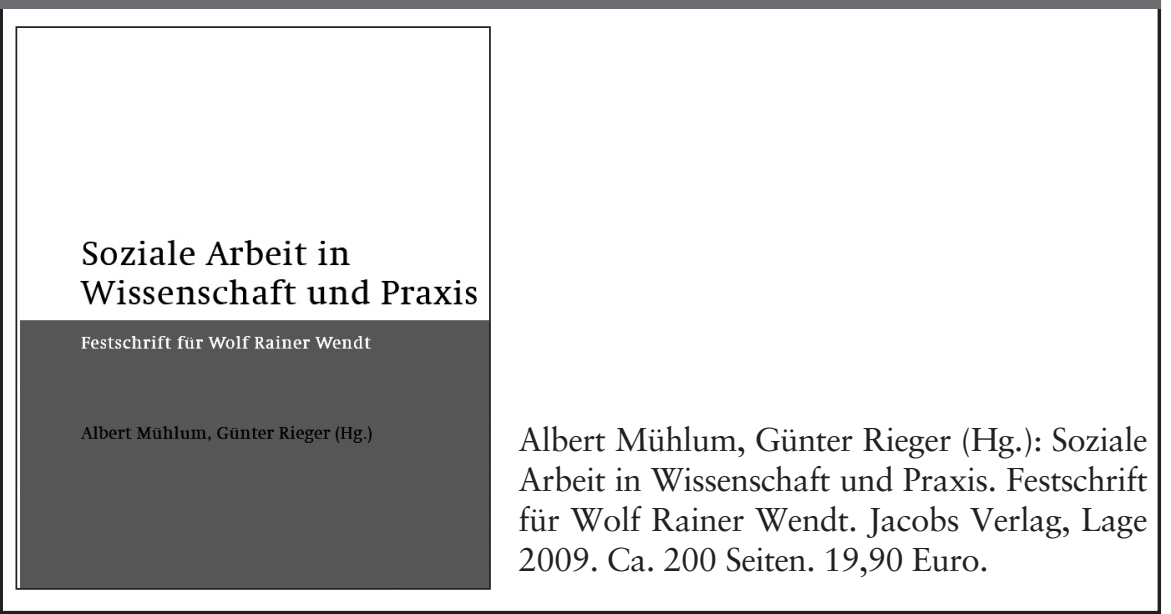

möglicher professioneller Unterstützung zu bessern.

Wendt argumentiert immer aus einer persönlich gefestigten, wissenschaftlich erarbeiteten Haltung heraus. Die Verknüpfung von Theorie und Praxis, das Individuum in seinen sozialen Bezügen, das Haushalten, Wirtschaften und Managen prägen Person und Werk. Damit wurde Wendt zu einer der bedeutendsten Köpfe der Sozialen Arbeit in der Bundesrepublik. Mit seiner großen »Geschichte der Sozialen Arbeit « (2 Bd.; 52008) trägt er viel zum Selbstverständnis der Sozialen Arbeit bei. Längst ist er selbst lebendiger Teil dieser Geschichte. Er hat die deutsche Sozialarbeit bereichert und Entscheidendes zu ihrer Professionalisierung beigetragen. Wir sind gespannt auf weitere Beiträge und wünschen nur das Beste.

\section{Expertenwissen für Führungskräfte}

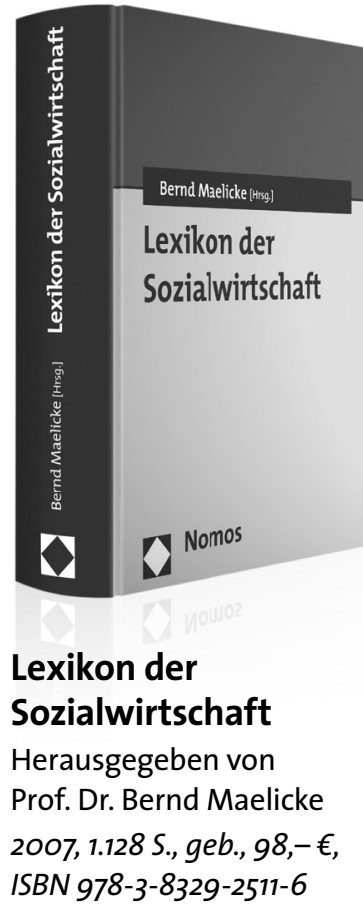

Das Werk umfasst ca. 700 Stichworte aus den einschlägigen Fachdisziplinen Betriebswirtschaft, Volkswirtschaft, Sozialpolitik, New Public Management, Recht, Erziehungswissenschaften, Sozialarbeit/ Sozialpädagogik und Psychologie.

Die über 130 Autoren und Autorinnen sind nationale und internationale Experten in Theorie und Praxis der Sozialwirtschaft. Das Werk systematisiert interdisziplinär die einschlägigen Fachbegriffe und stellt sie in den Zusammenhang der veränderten Anforderungen an die Handlungskompetenzen der Führungskräfte in der Sozialwirtschaft (Topmanagement, höheres und mittleres Management in sozialen Dienstleistungsorganisationen).

\section{Nomos}

Bitte bestellen Sie im Buchhandel ode versandkostenfrei unter $\$ www.nomos-shop.de 